\title{
Polyphenols from waste streams of food industry: valorisation of blanch water from marzipan production
}

\author{
Veronika Hellwig (D) Johanna Gasser
}

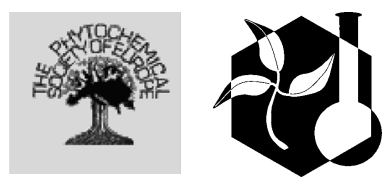

Received: 1 November 2019/Accepted: 14 February 2020/Published online: 22 February 2020

(C) The Author(s) 2020

\begin{abstract}
Waste fractions of food processing are promising sources of polyphenols, which are of high demand because of their favourable bioactivities. More recently, also wastewater and process water fractions are in focus of research and technologies for downstream processing, which is reviewed here. Adsorption as well as membrane technologies are widely used to achieve selective recovery of polyphenols from waste water. For technical implementation the processing of waste fractions must be separated from the primary food production process. Therefore, the key step is the efficient transfer of the waste fractions into a storable and transportable form of polyphenol-enriched fractions. This strategy is shown exemplarily for the marzipan production. Almond skin and blanch water are waste fractions containing catechin and procyanidins, for which a recycling concept has been developed. The polyphenolic ingredients of the blanch water can be specifically adsorbed by means of Amberlite resins or zeolites with high yield followed by ultrafiltration.
\end{abstract}

V. Hellwig $(\bowtie) \cdot$ J. Gasser

Technische Hochschule Lübeck - University of Applied Sciences, Mönkhofer Weg 239, 23562 Lübeck, Germany e-mail: veronika.hellwig@th-luebeck.de

\section{J. Gasser}

Institut für Organische und Analytische Chemie, Universität Bremen, Leobener Strasse 7 (NW2C), 28359 Bremen, Germany
Keywords Almond blanch water - Antioxidants . Downstream processing - Renewable resources . Waste water

$\begin{array}{ll}\text { Abbreviations } \\ \text { DPPH } & \text { 2,2-diphenyl-1-picrylhydrazyl } \\ \text { FRAP } & \text { Ferric reducing antioxidant power } \\ \text { HPLC } & \begin{array}{l}\text { High performance liquid } \\ \text { chromatography }\end{array} \\ \text { MAE } & \text { Microwave assisted extraction } \\ \text { ORAC } & \text { Oxygen radical absorbance capacity } \\ \text { PPO } & \text { Polyphenol oxidase } \\ \text { PVP } & \text { Polyvinylpyrrolidone } \\ \text { TE } & \text { Trolox equivalent } \\ \text { TEAC } & \text { Trolox equivalent antioxidant assay } \\ \text { TPC } & \text { Total phenolic content } \\ \text { VEREMA } & \text { Antioxidative und antibiotische } \\ & \text { Substanzen aus der stofflichen } \\ & \text { VErwertung von REststoffen der } \\ & \text { MArzipan-Industrie (engl. Antioxidant } \\ & \text { and antibiotic substances from material } \\ & \text { use of waste of the marzipan industry) }\end{array}$

\section{Introduction}

Polyphenols are highly demanded compounds as e.g. additives for functional food due to their antioxidant, antimicrobial and anti-inflammatory potential. More 
recently, the overall beneficial influence on the gut microbiota and by that on their metabolic activity is under investigation (Torres et al. 2017). The processing of polyphenol-containing waste streams from food processing represents a resource-efficient and sustainable source of polyphenol-enriched fraction. This review focuses on the methods for enrichment of polyphenols from waste water and process water, which enables upcycling of waste as well as recycling of water resources. Although there is extensive research on these issues, actual implementation in manufacturing facilities is often difficult. We briefly review the most commonly used technologies for recovery of polyphenols. Then we reflect the challenges and possible strategies on the example of recovery of polyphenols from almond blanch water to give suggestions for future projects.

\section{Waste streams from food production as source of polyphenols}

Waste streams from food production are a relevant source for secondary metabolites with bioactive profile in general (Dimou et al. 2019) and, in particular for polyphenols due to the following considerations: Amongst others, plants use polyphenolic compounds with antioxidative or antimicrobial bioactivity for protection. For photoprotection of plant cells, polyphenols are accumulated to form a shield under the epidermal layer (Sharma et al. 2019). The outer layers of stone fruits (bot. drupes) and nuts serve to protect the inner pulp and seeds from injury and microbial degradation. This is made possible by a structural design which, on the one hand, involves mechanical strength given by biopolymers forming the scaffold for the outer layer and are degradable by very specific enzymes or harsh conditions only. On the other hand, chemical defence mechanisms are enabled by inclusion of bioactive secondary metabolites like polyphenols (Dardick and Callahan 2014).

Polyphenolic compounds often cause an astringent or bitter taste which is proposed to precipitation with salivary proteins (Rinaldi et al. 2012). Besides, the antioxidative colourless polyphenols can be themselves oxidised to coloured or polymeric products which could also be disadvantageous at least in industrial food products. Polyphenol oxidase (PPO) plays an important role in these reactions (Selvarajan et al. 2018). Hence the outer layer of edible plant material, which contains a large part of the polyphenols, often is removed within the food processing or before consumption.

Therefore, peels (e.g. citrus peel) and skins (e.g. almond skins) form typical waste streams of food processing containing a rather high proportion of polyphenols (Monagas et al. 2007). The peeling is carried out using mechanical aids, but also enzymatically or by blanching with hot water. Due to their water solubility, many polyphenols are transferred at least partially into the aqueous phase. These process waters are additional waste streams from which polyphenols can be obtained. A prominent example of such an aqueous polyphenol-containing waste fraction is the olive oil mill (vegetation) water (Cassano et al. 2016). Here a downstream processing of the waste water is not primarily aimed at the recovery of polyphenols, but at the detoxification of water resources.

Table 1 gives an exemplary overview of solid as well as aqueous waste fractions of food production with a relevant proportion of polyphenols.

In former times, such waste fractions occurred at regional level and thereby in small-scale batches of varying composition. Due to industrialization of food production, not only the food products themselves, but also the associated waste fractions incurred in larger quantities of reproducible composition. Therefore, and going along with the demand for changeover from fossil to renewable resources as raw material base in general, waste fractions in the food industry are considered to have great potential not only for energetic use, but also for material use. Though many food companies are interested in upgrading their waste, they need to focus on their core business because of their size and organization. Therefore, a processing of waste fractions in the primary production unit is not appropriate in general.

\section{Screening of polyphenol content and antioxidant capacity}

Methods for the fast and reliable determination of the polyphenol content as a sum parameter as well as for analysing the polyphenol HPLC profile are used to evaluate the potential of waste fractions and to secure reproducibility of the new product in a technical scale. 
Table 1 Selective recovery of polyphenols from waste water of food industry (examples)

\begin{tabular}{lll}
\hline Source & Processing method & References \\
\hline Almond blanch water & See text & See text and cited references \\
Apple pomace extracts & Nanofiltration & Uyttebroek et al. (2018) \\
Blueberry pomace extract & Nanofiltration & Avram et al. (2017) \\
Nejayote (Nixtamalization wastewaters, corn industrial & Integrated membrane process & Castro-Muñoz and Yáñez- \\
wastewater) & & Fernández (2015) \\
Olive mill waste water & Nanofiltration & Alfano et al. (2018) \\
& Polymer incompatibility & Hajji et al. (2014) \\
& Activated carbon coated with milk & Yangui and Abderrabba (2018) \\
Pomegranate juice & proteins & Conidi et al. (2017) \\
Rose oil distillation wastewater & Ultra-/nanofiltration & Rusanov et al. (2014) \\
Winery wastewaters & Adsorption resin & Giacobbo et al. (2015) \\
& Microfiltration & Nayak et al. (2018) \\
\hline
\end{tabular}

The Folin Ciocalteu assay is widely used for determination of the total phenolic content (TPC) in natural product samples as well as in food supplements. The blue complex formed in electron transfer reactions between the reagent molybdotungstophosphoric heteropolyanion $\mathrm{PMoW}_{11} \mathrm{O}_{40}{ }^{4-}$ and phenolic compounds is used for photometric measurements at $\lambda_{\max }$ $=765 \mathrm{~nm}$ (Prior et al. 2005). The method is simple and sensitive, but lacks specificity because the reaction can be disturbed by other reducing substances in the sample. Results are reported most often as gallic acid equivalents, but also as catechin equivalents in flavonoid rich material.

Several in vitro methods are commonly in use to estimate antioxidant activity addressing different mechanisms. The ORAC assay (Oxygen Radical Absorbance Capacity) measures the kinetic of oxidative degradation of a fluorescent reagent by free radical generators in presence of the sample. Antioxidants in the sample are considered to decelerate the oxidative degeneration by protecting the fluorescent molecule via a free radical scavenging mechanism. Results are reported as equivalents of the antioxidant trolox (vitamin E analogue) (TE). The data can only be compared within test series. As there is no in vivo proof for the free-radical theory, the test is no longer used for determination and rating of antioxidant strength of food samples, but often still for comparison of antioxidant capacity in extracts and fraction. Other test systems are based on the reduction of persistent radicals (DPPH; TEAC) or of inorganic oxidising species (FRAP, Folin-Ciocalteu) and measurement of the samples ability to reduce metals (Pérez-Jiménez et al. 2008). It is recommended to match the results of at least two antioxidant assay systems to evaluate the antioxidant activity of mixtures. Recently, novel assay systems are developed for polyphenols and other chain-breaking antioxidants. Chain-breaking antioxidants react with radicals to form products which do not propagate the radical chain, so that autooxidation steps are impaired. Amorati and Valgimigli (2018) review recent developments of assay systems based on inhibited autooxidations. However, on the level of waste fractions as raw material, the interference by further compounds needs to be carefully considered.

\section{Processing of waste streams for recovery of polyphenol fraction}

The first generation of waste streams projects from food industry focussed on solid waste fractions. Meanwhile, more attention is also paid to process water as waste fractions. Such waste fractions are in focus of this summary. A rather high volume of the waste fraction in production scale is often going along with a rather low concentration of polyphenols. Anyhow the regular volume of process or waste water fractions is too large in industrial processes to seriously consider the transport of the unprocessed 
waste water to the secondary downstream processing unit. In the case of olive mill waste water (see Table 1) the elimination of polyphenols from process water is very relevant due to the rather high toxicity of olive mill wastewater and due to the needed reusability of water.

Therefore, efficient and smooth enrichment steps are to be implemented. Especially for process water, enrichment is technically spoken often mainly in the meaning of volume reduction. In this mini-review the recovery and concentration of polyphenols by adsorption and membrane processes is briefly summarized due to their rather good energy-efficiency and feasibility for upscaling. In addition, these two steps are consistent with the planned use of the polyphenols in food additives industries. For the examples in Table 1 also technology of processing is given.

In fact, food-grade adsorbents are already present here, e.g. known from the beverage industry.

Adsorption on suitable material is an already established efficient method in food industry to deplete polyphenols from aqueous solutions. Hereby the slightest influence on the product has to be avoided. Various amorphous materials on the basis of ion-exchange resins or polymers are long-term used. Polyvinylpyrrolidone (PVP) is used as fining agent in beers to avoid haze effects due to polyphenolprotein-interactions. They are also commonly used in adsorption processes for environmental applications, e.g. wastewater treatment. However, recovery of polyphenols from waste fractions must be compatible with further application especially in food industry and should be efficient concerning the secondary product. Regarding the selective recovery of polyphenols from water fractions by adsorption, resin materials are best studied. A broad variety of synthetic polymer adsorbents on the basis of polystyrenedivinylbenzene copolymers or divinylbenzeneethylvinylbenzene copolymers is nowadays available. Adsorption on resins is a commonly used separation technology as the relatively inexpensive resins are durable and chemical stable, thus harmless regarding toxicity. The adsorption steps can be performed efficient due to high adsorption capacity and selectivity and ease of regeneration.

Other materials include activated charcoals, e.g. for the recovery of flavonoids from aqueous solutions with the disadvantage, that partial irreversible adsorption diminishes the yield of polyphenols. Activated charcoals themselves are often produced from agricultural by-products themselves. Haddad et al. (2017) describe the valorisation of olive mill water by impregnation on sawdust. Recently, also beta-zeolites are found to be promising adsorbents for recovery of polyphenols from renewable resources (Thiel et al. 2013).

Advantages of membrane techniques are their minimal space and energy requirements. Membranes separate different components of a solution due to their particle sizes or other properties that affect permeability. The treatment in a membrane process separates the wastewater into two phases: the permeate passes through the membrane while the retentate (sometimes referred to as the concentrate) remains on the feed side of the membrane. For enrichment of polyphenols a pressure gradient is used to power the mass transfer process resulting in filtration steps ranging from ultrafiltration (macromolecules with 1-300 kDa, 2-10 bar) or nanofiltration (small molecules with 350-1000 Da, 10-40 bar). Casas et al. (2017) reviewed a broad range of application both of adsorption as well as of membrane technology for recovery of polyphenols. Processing of natural products (Castro-Muñoz and Fila 2018) and agro-food byproducts (Cassano et al. 2018) by membrane technologies for the recovery of high-added value compounds as polyphenols are reviewed.

Both the adsorption step and the membrane technique cope with the challenge to preferably separate the upcycling process from the primary production. Deactivation of adsorber surface by non-polyphenolic material is a typical issue during upscaling.

The authors are aware that there are a lot of lab scale separation and enrichment techniques like prep HPLC which are perfect for isolation of single polyphenols in lab scale, but often not feasible for an upscaling. Fractionation of mixtures of polyphenols is often reported by countercurrent chromatography techniques, e.g. HSCCC. This type of separation technique does not employ any solid (supporting) matrix. For a summary see Winterhalter and Engelhardt (2017).

\section{Example: valorisation of blanch water in marzipan production}

In industrial marzipan production sites many tons of marzipan are produced on a daily basis. Consequently, 
in the here sampled production site up to $10 \mathrm{t}$ of almonds are processed daily. The almond skins contain a crude mixture of polyphenols with high structural variety (up to $30 \%$ polyphenols; Brieskorn and Betz 1988) to protect the almond kernel with catechin and epicatechin as well as their oligomers (procyanidins) as main metabolites showing promising bioactivities (see Fig. 1). In Bolling (2017) all polyphenols from almond are summarized. Besides the already mentioned proanthocyanidins, flavonoids and their glycosides (e.g. isorhamnetin-3-O-rutinosid and kempferol-3-O-ruinoside) as well as phenolic acids and aldehydes (e.g. hydroxybenzoic acids and hydroxybenzaldehydes) and other phenolics (e.g. isoflavones, stilbenes and lignans) are described. Therefore, the valorisation of the waste streams of marzipan industry by recovery of the antioxidant polyphenols is the aim of the VEREMA project in cooperation with the food industry beneath others (e.g. Prgomet et al. 2017; Smeriglio et al. 2016). The first step in the marzipan production is the blanching of the almonds in hot water $\left(96{ }^{\circ} \mathrm{C}\right)$ to peel off the skin. Regarding the recovery of polyphenols this blanching step is also an important selective extraction step of polyphenols. Already Hughey et al. (2012) described, that during peeling by blanching about $73 \%$ of the almond polyphenols leach to the blanch water. So, the blanch water (approx. 5-10 $\mathrm{m}^{3} /$ day in the sampled site) is the second waste stream besides the almond skin containing the polyphenols with good water solubility, while other polyphenols remain in the skins. An important limitation, which is typical for projects aiming at valorisation of waste stream in food industry, is the fact that the primary production process must not be affected. The valorisation concept is therefore based on transfer of large volume waste fractions into a transportable and storable form including selective enrichment steps by adsorption and ultrafiltration technology.

In lab scale the processing of blanch water is performed in 301 batches (see Fig. 2). In the technical realization a continuous process is to be established. After sampling the blanch water still contains solids, so in the lab scale the blanch water is filtered to protect the disc stack separator, which is used in the next step. In the technical process there is a settling tank. After disc stack separation approx. $2.3 \mathrm{~g}$ gallic acid equivalents per 1 blanch water are determined (according to ISO 14502-1:2005 2005). The clear solution is subjected to an adsorption step on Amberlite XAD-7HP (Buran et al. 2014). After elution with acetone and evaporation around min. $80 \%$ of gallic acid equivalents of the original blanch water is recovered and available as an enriched phenolic extract. In the technical realization the adsorption step should be performed by inserting the adsorption material after the settling tank to avoid affecting the primary production process. The polyphenol loaded adsorber

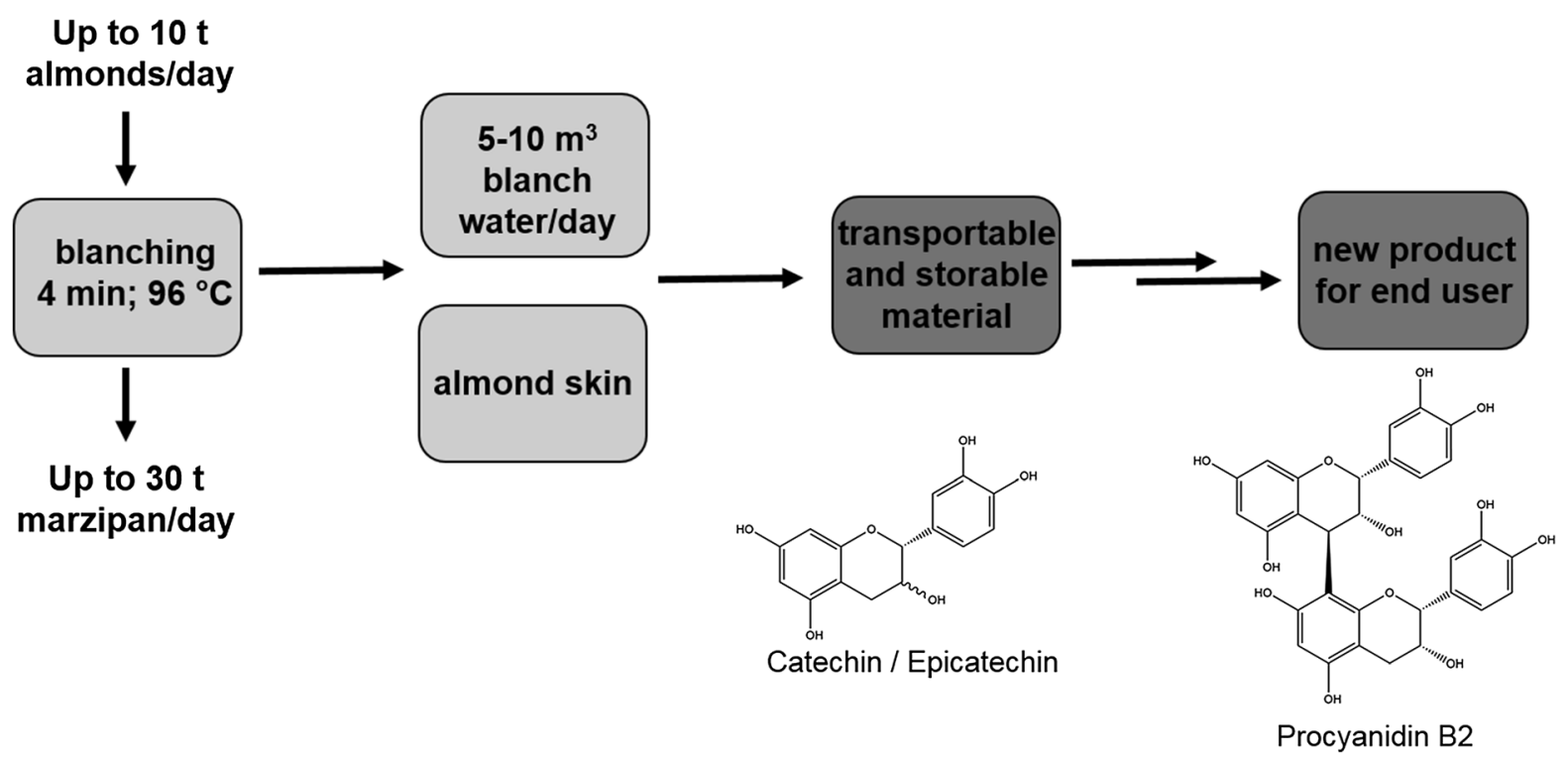

Fig. 1 Valorisation of waste streams in marzipan industry (VEREMA project) 


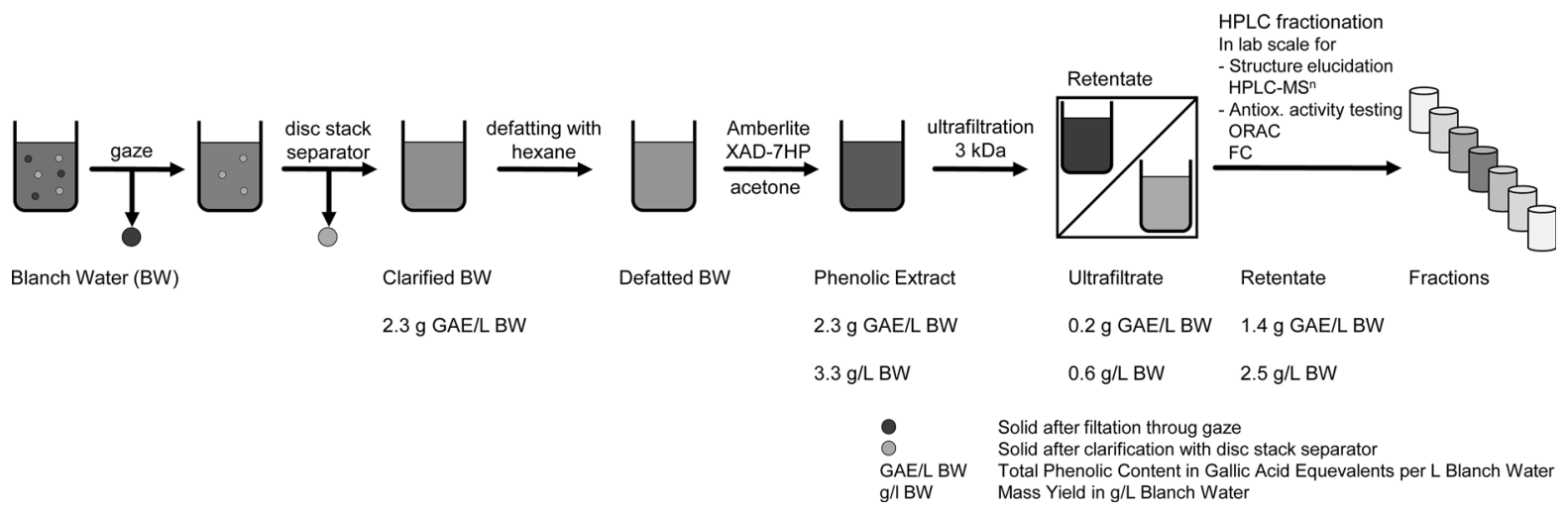

Fig. 2 Processing of almond blanch water by adsorption on resin material and ultrafiltration

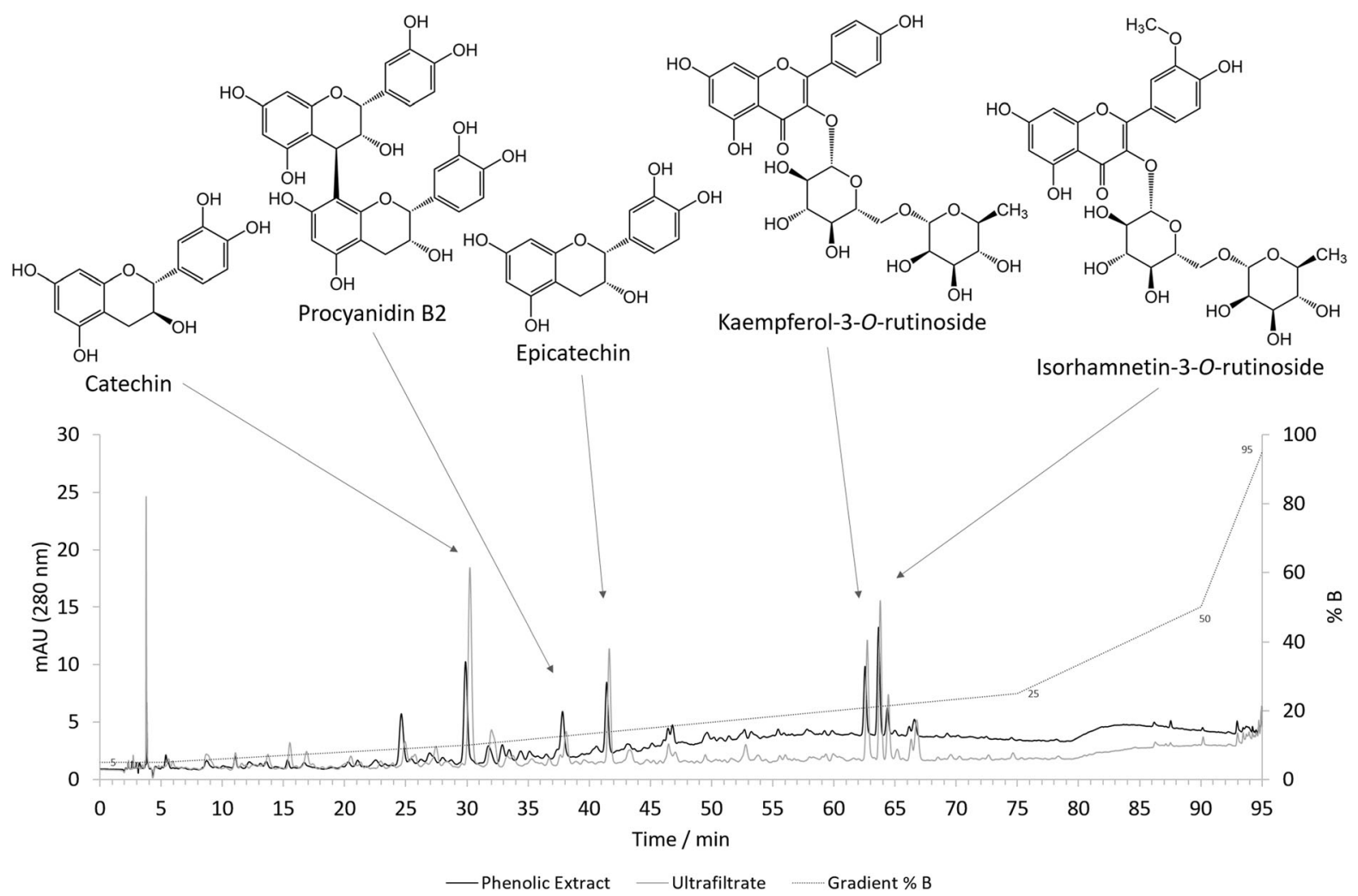

Fig. 3 HPLC chromatogram of blanch water, phenolic extract and ultrafiltrate (LiChrosorb $\mathrm{C}_{18} \mathrm{H}_{2} \mathrm{O} /$ Acetonitrile/HOAc), assignment via $\mathrm{MS}^{\mathrm{n}}$ and comparison with analytical standards

material can then be transported to a conventional downstream facility, while the depleted process water is disposed of as usual. In the further processing importance to flexibility to either create material containing the low molecular weight catechins or the oligomers or the polymers was attached. If applicable, an ultrafiltration step through a membrane (cut-off $3 \mathrm{kDa}$ ) was performed.

For determination of the chemical structures, their biological properties as well as the recovery potential of the individual substances the polyphenol fraction is chromatographed (preparative scale HPLC, C18 HTec, acetonitrile/water/acetic acid, see Fig. 3). 
Structural characterization is performed by ion trap $\mathrm{MS}^{\mathrm{n}}$ (Bruker amazon X) and comparison with commercially available analytical standards. Total phenolic content was measured with Folin Ciocalteu assay, while oxygen radical capacity assay was used to determine the antioxidant activity of the fractions.

To analyse the substances remaining in the almond skin after blanching, almond skins were extracted by MAE (Valdés et al. 2015) followed by concentration of the polyphenolics via SPE.

Testing of various polymeric adsorbents as well as mineral adsorbents resulted in Amberlite XAD7HP and Clariant beta zeolites as suitable materials for polyphenol adsorption in blanch water. Effective desorption is achieved with acetone or ethanol, respectively. Due to the different structural characteristics different extract profiles are yielded. While the used Amberlite resin has a large surface with a macroreticular structure, the zeolites have smaller pores. Mixtures of different sizes of polyphenols are obtained with the XAD resin. With the zeolites only the smaller oligomers were yielded. The larger oligomers could be recovered by Amberlite in a second cartridge.

\section{Concluding remarks}

These results should lead to a scale up of the described methods for polyphenol recovery in food industry for utilization of economically usable fractions for HealthCare, cosmetics and food production. Our concept for material use is based on the possibility of simply transferring the residual fractions into a transportable and possibly storable form. Adsorption step enables separation of the upcycling process from the production. Application of specific adsorber materials enrich certain subgroups of polyphenols. This allows for not location-bound processing in a conventional downstream facility. In addition, the methodology can be converted to similar processes in food technology. Preliminary experiments have shown that the described methodology can also be applied for recovery of polyphenols from aqueous residues of other food industries. We tested the recovery of flavonoids from aqueous bottom products of pectin production based on citrus peels as well as the removal of phenolic ingredients from olive mill waste water. The next step will be to evaluate if pooling of polyphenol extract material of different origin is feasible. The main economic risk of waste valorisation projects from food industry is that the production of bioactive substances is technically feasible, but economically not viable.

Acknowledgements Open Access funding provided by Projekt DEAL. The project VEREMA was supported by a Grant from the German Federal Ministry of Education and Research BMBF 13FH018IX5. The authors are grateful to PD Dr. Ralf Moll for scientific support as well as to Fabio Haeseler, Rouven Smolin, Ulrike Schefe and Eva Ziehm at the Center of Industrial Biotechnology (CIB) at TH Lübeck for technical assistance. The supervision of the PhD thesis of J.G. by Prof. Dr. Peter Spiteller, Universität Bremen is gratefully acknowledged.

Open Access This article is licensed under a Creative Commons Attribution 4.0 International License, which permits use, sharing, adaptation, distribution and reproduction in any medium or format, as long as you give appropriate credit to the original author(s) and the source, provide a link to the Creative Commons licence, and indicate if changes were made. The images or other third party material in this article are included in the article's Creative Commons licence, unless indicated otherwise in a credit line to the material. If material is not included in the article's Creative Commons licence and your intended use is not permitted by statutory regulation or exceeds the permitted use, you will need to obtain permission directly from the copyright holder. To view a copy of this licence, visit http://creativecommons.org/licenses/by/4.0/.

\section{References}

Alfano A, Corsuto L, Finamore R et al (2018) Valorization of olive mill wastewater by membrane processes to recover natural antioxidant compounds for cosmeceutical and nutraceutical applications or functional foods. Antioxidants $7: 72$

Amorati R, Valgimigli L (2018) Methods to measure the antioxidant activity of phytochemicals and plant extracts. J Agric Food Chem 66:3324-3329

Avram AM, Morin P, Brownmiller C et al (2017) Concentrations of polyphenols from blueberry pomace extract using nanofiltration. Food Bioprod Process 106:91-101

Bolling BW (2017) Almond polyphenols: methods of analysis, contribution to food quality, and health promotion. Compr Rev Food Sci Food 16:346-368

Brieskorn CH, Betz R (1988) Polymere procyanidine, die prägenden Bestandteile der Mandel-Samenschale. Z Lebensm Unters Forsch 187:347-353

Buran TJ, Sandhu AK, Li Z et al (2014) Adsorption/desorption characteristics and separation of anthocyanins and polyphenols from blueberries using macroporous adsorbent resins. J Food Eng 128:167-173

Casas MP, Soto ML, Diaz-Reinoso B et al (2017) Purification of polyphenolic extracts from natural sources-adsorption 
and membrane technologies. In: Cuevas-Valenzuela $\mathrm{J}$, Vergara-Salinas JR, Pérez-Correa JR (eds) Advances in technologies for producing food-relevant polyphenols, contemporary food engineering, vol 39. Taylor \& Francis, Boca Raton, pp 221-259

Cassano A, Conidi A, Galanakis CM et al (2016) 7-recovery of polyphenols from olive mill wastewaters by membrane operations. In: Figoli A, Cassano A, Basile A (eds) Membrane technologies for biorefining. Woodhead Publishing, Sawston

Cassano A, Conidi C, Ruby-Figueroa R et al (2018) Nanofiltration and tight ultrafiltration membranes for the recovery of polyphenols from agro-food by-products. Int J Mol Sci 19:351

Castro-Muñoz R, Fila V (2018) Membrane based technologies as an emerging tool for separating high-added-value compounds from natural products. Trends Food Sci Technol 82:8-20

Castro-Muñoz R, Yáñez-Fernández J (2015) Valorization of nixtamalization wastewaters (Nejayote) by integrated membrane process. Food Bioprod Process 95:7-18

Conidi C, Cassano A, Caiazzo F et al (2017) Separation and purification of phenolic compounds from pomegranate juice by ultrafiltration and nanofiltration membranes. Int $\mathbf{J}$ Mol Sci 195:1-13

Dardick C, Callahan AM (2014) Evolution of the fruit endocarp: molecular mechanisms underlying adaptations in seed protection and dispersal strategies. Front Plant Sci 5:284

Dimou C, Karantonis HC, Skalkos D et al (2019) Valorization of fruits by-products to unconventional sources of additives, oil, biomolecules and innovative functional foods. Curr Pharm Biotechnol 20:776

Giacobbo A, Matosdo Prado J, Meneguzzi A et al (2015) Microfiltration for the recovery of polyphenols from winery effluents. Sep Purif Technol 143:12-18

Haddad K, Jeguirim M, Jerbi B et al (2017) Olive mill wastewater: from a pollutant to green fuels, agriculturalwater source and biofertilizer. ACS Sustain Chem Eng 5:8988-8996

Hajji F, Kunz B, Weissbrodt J (2014) Polymer incompatibility as a potential tool for polyphenol recovery from olive mill wastewater. Food Chem 156:23-28

Hughey CA, Janusziewicz R, Minardi CS et al (2012) Distribution of almond polyphenols in blanch water and skins as a function of blanching time and temperature. Food Chem $131: 1165-1173$

ISO 14502-1:2005 (2005) Determination of substances characteristic of green and black tea-part 1: content of total polyphenols in tea-colorimetric method using FolinCiocalteu reagent, Last reviewed and confirmed in 2015

Monagas M, Garrido I, Lebrón-Aguilar R et al (2007) Almond (Prunus dulcis (Mill.) D.A. Webb) skins as a potential source of bioactive polyphenols. J Agric Food Chem 55:8498-8507

Nayak A, Bhushan B, Rodriguez-Turienzo L (2018) Recovery of polyphenols onto porous carbons developed from exhausted grape pomace: a sustainable approach for the treatment of wine wastewaters. Water Res 145:741-756

Pérez-Jiménez J, Arranz S, Tabernero M et al (2008) Updated methodology to determine antioxidant capacity in plant foods, oils and beverages: extraction, measurement and expression of results. Food Res Int 41:274-285

Prgomet I, Gonçalves B, Domínguez-Perles R et al (2017) Valorization challenges to almond residues: phytochemical composition and functional application. Molecules 22(10): 1774

Prior RL, Wu X, Schaich K (2005) Standardized methods for the determination of antioxidant capacity and phenolics in foods and dietary supplements. J Agric Food Chem 53:4290-4302

Rinaldi A, Gambuti A, Moio L (2012) Application of the SPI (Saliva Precipitation Index) to the evaluation of red wine astringency. Food Chem 135:2498-2504

Rusanov K, Garo E, Rusanova M et al (2014) Recovery of polyphenols from rose oil distillation wastewater using adsorption resins-a pilot study. Planta Med 80(17):1657-1664

Selvarajan E, Veena R, Manoj Kumar N (2018) Polyphenol oxidase, beyond enzyme browning. In: Singh J, Sharma D, Kumar G, Sharma N (eds) Microbial bioprospecting for sustainable development. Springer, Singapore

Sharma A, Shahzad B, Rehman A et al (2019) Response of phenylpropanoid pathway and the role of polyphenols in plants under abiotic stress. Molecules 24(13):2452

Smeriglio A, Mandalari G, Bisignano C et al (2016) Polyphenolic content and biological properties of avola almond (Prunus dulcis Mill. D.A. Webb) skin and its industrial byproducts. Ind Crop Prod 83:283-293

Thiel A, Tippkötter N, Suck K et al (2013) New zeolite adsorbents for downstream processing of polyphenols from renewable resources. Eng Life Sci 13(3):239-246

Torres JL, Ramos-Romero S, Pérez-Jiménez J (2017) Key aspects of polyphenols and health-metabolic fate, mechanisms of action, and influence of gut microbiota. In: Cuevas-Valenzuela J, Vergara-Salinas JR, Pérez-Correa JR (eds) Advances in technologies for producing foodrelevant polyphenols, contemporary food engineering, vol 39. Taylor \& Francis, Boca Raton, pp 221-259

Uyttebroek M, Vandezande P, Van Dael M (2018) Concentration of phenolic compounds from apple pomace extracts by nanofiltration at lab and pilot scale with a techno-economic assessment. J Food Process Eng 41:e12629

Valdés A, Vidal L, Beltrán A et al (2015) Microwave-assisted extraction of phenolic compounds from almond skin byproducts (Prunus amygdalus): a multivariate analysis approach. J Agric Food Chem 63:5395-5402

Winterhalter P, Engelhardt UH (2017) Fractionation of Polyphenols. In: Cuevas-Valenzuela J, Vergara-Salinas JR, Pérez-Correa JR (eds) Advances in technologies for producing food-relevant polyphenols, contemporary food engineering, vol 39. Taylor \& Francis, Boca Raton, pp 221-259

Yangui A, Abderrabba M (2018) Towards a high yield recovery of polyphenols from olive mill wastewater on activated carbon coated with milk proteins: experimental design and antioxidant activity. Food Chem 262:102-109

Publisher's Note Springer Nature remains neutral with regard to jurisdictional claims in published maps and institutional affiliations. 